\title{
UhJMSR
}

Journal of Medical and Surgical Research

\section{PSYCHOMETRIC PROPERTIES OF THE NURSE CULTURAL COMPETENCE SCALE AMONG FILIPINO NURSES}

\author{
Gil P. Soriano
}

College of Nursing, San Beda University, Manila, Philippines

Graduate School, Wesleyan University-Philippines, Cabanatuan City, Nueva Ecija, Philippines

\begin{abstract}
Purpose: Assessment of the cultural competence of nurses is becoming an important parameter in assessing the provision of quality care to patients especially in multicultural populations such as the Philippines. The study was conducted to assess the construct validity and internal consistency reliability of the Nurse Cultural Competence Scale (NCCS) among Filipino nurses. Methods: A methodological study was utilized and a convenience sample of 124 Filipino nurses were included in the study. An exploratory factor analysis was used to assess the construct validity of the instrument while internal consistency reliability was determined using Cronbach's $\alpha$ coefficient and item-total correlations. Results: The 20-item NCCS was loaded on one dimension with factor loadings ranging from 0.72 to 0.95 , explaining $79.19 \%$ of the total variance. Cronbach's $\alpha$ was 0.986 which demonstrated high reliability and item-total correlations were between 0.811 and 0.929 . Conclusion: The NCCS is a reliable and valid instrument for the measurement of cultural competencies among Filipino nurses.
\end{abstract}

Keywords: Cultural competence; Filipino nurses; Reliability; Validity.

\section{Corresponding Author:}

Gil P. Soriano, MHPEd, RN.

Address: College of Nursing, San Beda University, 638 Mendiola St., San Miguel, Manila

E-mail: gil.p.soriano@gmail.com

ORCID: 0000-0002-6349-5560

Copyright (C) 2012- 2021 Gil P. Soriano. This is an open access article published under Creative Commons Attribution -Non Commercial- No Derives 4.0 International Public License (CC BY-NC-ND). This license allows others to download the articles and share them with others as long as they credit you, but they can't change them in any way or use them commercially.

doi: 10.46327/msrjg.1.000000000000----

*****Published in 2021. doi url: https://doi.org/10.46327/msrjg

\section{INTRODUCTION}

There is an ever-growing concern regarding cultural diversity in healthcare settings. Studies have shown that those from racially and ethnically diverse populations suffer from higher rates of illness and disability and have reduced access to health care as compared to the overall population [1-2]. Hence, the provision of care that is appropriate and culturally sensitive to all patients becomes a necessity [3]. This has been supported by international nursing literatures as they emphasized the need for culturally competent care [4-5].

Culturally competent care is defined as the care that is responsive and reactive to the diversity of the patient population and cultural factors that can affect health and healthcare, such as languages, communication styles, beliefs, attitudes, and behaviors [6]. Nurses, in particular, should be encouraged to understand more about the cultural needs of their patients and to provide culturally appropriate treatment [7-9]. This is due not only to the fact that registered nurses make up the largest group of healthcare workers, accounting for more than half of the global health workforce [10], but also to the fact that they devote more time personally caring for patients than all other members of the healthcare team [11].

Cultural competence is commonly seen as a foundational pillar for reducing health disparities through the delivery of culturally sensitive and unbiased quality care [12]. It was also shown to improve patient satisfaction and health outcomes [13]. Given its importance, the challenge in measuring cultural competence among nurses should be addressed.

The development of instruments related to the cultural competence qualities of health care professionals rather than patient impressions of their care or health outcomes has facilitated the assessment of cultural competence in nursing practice and education [5]. Several researches have emphasized that a valid measurement tool is necessary in order to assess the cultural 
competencies of nurses [14-15]. For this reason, Perng and Watson [16] developed the Nurse Cultural Competence Scale (NCCS) using the Mokken scale. It is consists of twenty items that evaluates the cultural skills, cultural knowledge, and cultural sensitivity of nurses.

The nurses' cultural competence is difficult to determine; however, with the changing demographics in the Philippines, assessing quality care for individuals in different groups has become an increasingly important issue. However, to the best of my knowledge, there has been no studies conducted yet which ascertain the validity and reliability of an instrument in determining the cultural competence of Filipino nurses. Hence, this study was conducted in order to assess the construct validity and internal consistency reliability of the Nurse Cultural Competence Scale (NCCS) among Filipino nurses.

\section{METHODS}

\section{Design and Participants}

This methodological study was conducted between the period of January to March 2019 and recruited Filipino nurses working in medical-surgical wards in selected hospitals in Manila through convenience sampling. The inclusion criteria included nurses who voluntarily participated in the study. The sample size was estimated based on the criterion than at least 5 participants per item were needed for conducting an explanatory factor analysis [17]. Consequently, since there were a total of 20 items in the instruments consisting of three sub-scales, the study needed to include 100 participants. One hundred fifty questionnaires were given to nurses for determining the final sample size. A total of one hundred twenty four nurses returned their questionnaires, which has a response rate of $83 \%$. Further, since all of the participants can understand the English language, backward and forward translation were not done and content validity index was not checked.

\section{Research Instrument}

Demographic Information Questionnaire. The questionnaire forms involving demographic characteristics of the participants which includes age, sex, and length of service as a nurse in the hospital.

Nurse Cultural Competence Scale. NCCS is a 20 item tool consisting of three subscales which includes cultural knowledge, cultural sensitivity, and cultural skills. Twelve statements measure cultural skills, six statements measure cultural knowledge, and two statements measure cultural sensitivity. The statements are rated using a Likert scale with agreement levels of strongly disagree (1), disagree (2), not sure (3), agree (4), and strongly agree (5); ratings may vary between 20 and 100. A higher rating indicates a high cultural competence. The original form of the scale was prepared using Mokken scale analysis with Cronbach's alpha reliability coefficient of 0.96 [16].

\section{Ethical Consideration}

Approval from the Ethics Review Board of Arellano University was secured in order to conduct the study.

\section{Data Analysis}

Data management and statistical analyses were conducted by using the SPSS version 21.0 (IBM Corporation, Armonk, NY). Demographic characteristics were analyzed using descriptive statistical analyses. Internal consistency using Cronbach's alpha coefficient and item-total correlations were measured to assess the reliability of NCCS among Filipino nurses. An item-total correlation higher than 0.30 [18] and Cronbach's alpha coefficient of more than 0.70 [19] were the required criteria.

In order to assess the construct validity of the instrument, an exploratory factor analysis was performed. Specifically, principal component analysis method with promax rotation was performed and factors with eigenvalue of more than 1.0 was used to examine the factors in each subscale. The adequacy of the samples was measured using Kaiser-Meyer-Olkin (KMO) test and the recommended value was 0.60 [20]. In order to assume factorability of correlation matrix, Bartlett's test of sphericity must be significant at $\alpha<0.05$ [21] while factor loading coefficient of 0.30 and higher was accepted as the criteria to retain an item in a scale [22].

\section{RESULTS}

\section{Demographic characteristics}

The mean age of the respondents was $29.37( \pm 6.94)$ years old and the majority of the respondents were female $(80.65 \%)$. On the other hand, the mean length of service of the respondents was 4.55 years $( \pm 5.91)$ (Table I) 


\section{YhJMSR}

Journal of Medical and Surgical Research

Table I: Demographic characteristics of the participants $(\mathrm{n}=124)$

\begin{tabular}{|c|c|c|c|}
\hline Profile & $\mathrm{n}$ & $\%$ & Mean (SD) \\
\hline Age & & & $29.37 \quad(6.94)$ \\
\hline Gender & & & \\
\hline Male & 24 & 19.35 & \\
\hline Female & 100 & 80.65 & \\
\hline Length of Service & & & $(5.91)$ \\
\hline
\end{tabular}

\section{Reliability}

The item mean of the scale, standard deviation, itemtotal correlation and Cronbach alpha coefficients were determined. The corrected item-total correlation of each item varied between 0.81 and 0.92, and the Cronbach's coefficient of the 20-item NCCS was found to be 0.986 (Table II).

Table II. Factor Loadings, Item Analysis, and Item-Total Correlations for the NCCS

Factor Loading Item Mean
(SD) Item-Total Correlation

similarities of diverse cultures.

2. I can teach and guide colleagues about planning nursing interventions for patients from different cultures

3. I can use examples to illustrate communication skills with patients from different cultures

4. I can teach and guide colleagues about the communication skills for patients from different cultures.

5. I can explain the influences of cultural factors on one's beliefs/ behavior toward health/illness to patients from diverse ethnic groups.

6. To me, collecting information on each patient's beliefs/behavior about health/illness is very easy

7. I can teach and guide colleagues about the cultural knowledge of health and illness.

8. I can teach and guide colleagues to display appropriate behavior, when they implement nursing care for patients from different cultures

9. I am familiar in health and/or illness related cultural knowledge and/or theory.

10. I can explain the influence of culture on a patient's beliefs/behavior about health/illness.

11. I can list the methods or ways of collecting health, illness, and cultural-related information

12. I can compare health/illness beliefs among patients from different cultures

13. I can easily identify the care needs of patients from different cultures

14. When implementing nursing activities, I can fulfil the needs of patients from different cultures

15. I can explain the possible relationships between the health/illness beliefs and culture of patients

16. I can establish nursing goals according each patient's cultural background.

17. I usually actively strive to understand the beliefs of different cultural groups.

18. When caring for patients from different cultures, my behavioral response usually will not differ much from the patient's cultural norms.

19. I can employ the communication skills necessary for patients from different cultures

20. I usually discuss differences between the patient's health beliefs/behavior and nursing knowledge with each patient

\begin{tabular}{|c|c|c|c|}
\hline 0.81 & $3.35(1.16)$ & 0.82 & 0.985 \\
\hline 0.79 & $3.36(1.13)$ & 0.81 & 0.985 \\
\hline 0.81 & $3.33(1.17)$ & 0.83 & 0.985 \\
\hline 0.87 & $3.24(1.05)$ & 0.87 & 0.985 \\
\hline 0.88 & $3.22(0.99)$ & 0.88 & 0.985 \\
\hline 0.83 & $3.16(1.02)$ & 0.82 & 0.985 \\
\hline 0.92 & $3.23(1.03)$ & 0.92 & 0.985 \\
\hline 0.91 & $3.28(1.06)$ & 0.90 & 0.985 \\
\hline 0.86 & $3.16(0.90)$ & 0.86 & 0.985 \\
\hline 0.89 & $3.28(0.96)$ & 0.88 & 0.985 \\
\hline 0.88 & $3.15(0.95)$ & 0.87 & 0.985 \\
\hline 0.88 & $3.23(0.95)$ & 0.88 & 0.985 \\
\hline 0.89 & $3.21(1.01)$ & 0.88 & 0.985 \\
\hline 0.93 & $3.27(1.03)$ & 0.91 & 0.985 \\
\hline 0.92 & $3.23(0.99)$ & 0.91 & 0.985 \\
\hline 0.94 & $3.29(1.06)$ & 0.92 & 0.984 \\
\hline 0.88 & $3.19(1.11)$ & 0.86 & 0.985 \\
\hline 0.89 & $3.18(1.11)$ & 0.88 & 0.985 \\
\hline 0.92 & $3.28(1.08)$ & 0.91 & 0.985 \\
\hline 0.84 & $3.27(0.98)$ & 0.83 & 0.985 \\
\hline
\end{tabular}




\section{Construct Validity}

Explanatory factor analysis was performed to test the construct validity of the 20-item NCCS among Filipino nurses. Bartlett's test and KMO measure of sampling adequacy were carried out to ensure the characteristics of the data were proper in terms of the factor analysis. The results revealed that the KMO test was 0.963, and the Bartlett's test was 3668.765 (df= 190, $\mathrm{p}=0.000$ ). The items were loaded on one factor which explained $79.19 \%$ of the total variance. The twenty-item loaded on one factor ranged from 0.72 to 0.95 (Table 2).

\section{DISCUSSION}

The NCCS scale is an instrument that assesses a nurse's ability to provide holistic care to culturally patients. The NCCS scale has been validated in several countries where improving nurses' cultural competence is seen as a major obstacle in practice and vocational education.

The reliability of the NCCS among Filipino nurses showed an acceptable internal consistency $(\alpha=0.986)$ which implies that all the items are closely related to each other as one scale. This value is higher than the recommended Cronbach's alpha coefficient $(0.70)$ for an instrument [19]. Following this recommendation, the NCCS had a high internal consistency and was relatively appropriate for use among Filipino nurses. The internal consistency of the instrument was also comparable to the Cronbach's alpha of the Turkish version of the NCCS which showed a coefficient of 0.96 among 235 nurses [23], the Arabic version with alpha coefficient of 0.96 [24], the Spanish version with alpha coefficient of 0.95 [25], the Polish version with alpha coefficient of 0.94 among 238 nurses [26] and the Italian version with the alpha coefficient of 0.91 [27]. The most commonly recorded indicator of internal consistency in scale growth is Cronbach's $\alpha$. Higher Cronbach's alpha values suggest greater reliability and, as a result, greater measurement precision [28]. In addition, items in the NCCS among Filipino nurses demonstrated an acceptable corrected item-total correlations which is higher than 0.30 [18]. For the construct validity, an exploratory factor analysis using principal component analysis with Promax rotation was performed. The results revealed a KMO value of 0.963 and Bartlett's test of 3668.765 $(\mathrm{df}=190, \mathrm{p}=0.000)$. In order to ensure the adequacy of samples, a recommended value of 0.60 [20] was needed whereas correlation must be significant at $\alpha<$ 0.05 to assume factorability of matrix [21]. This satisfy the set criteria which indicates an excellent sampling adequacy and suitability for factor analysis. On the other hand, items in the instrument were all loaded into one dimension and have factor loadings ranging from 0.72 to 0.95 which showed an acceptable value of higher than 0.30 [22]. The Turkish version of the instrument has a factor loading ranging from 0.70 to 0.83 [23], the Arabic version had factor loadings ranged from 0.495 to 0.828 [24] while the Spanish version had factor loadings ranging from 0.490 to 0.780 [25] where all the items were also loaded in one dimension and showed acceptable factor loadings supporting the findings of this study. However, the original NCCS consists of three dimensions namely: cultural skills, cultural knowledge and cultural sensitivity with Mokken scale ranging from 0.49 to 0.74 [16] while the Polish version includes the factor structure in the original NCCS with the addition of the cultural awareness as its fourth structure [26].

\section{STUDY LIMITATIONS}

One of the limitations of the study is that convenience sampling was used and that the participants were recruited in Manila, which limits the generalizability of the results. Also, confirmatory factor analysis and other validity and reliability measure were not conducted, hence, including other measures in future studies is needed.

\section{CONCLUSION}

The NCCS was shown to be a valid and reliable instrument for assessing cultural competencies among Filipino nurses. Hence, this instrument can be provide nurses with efficient evaluation of their clinical competence. Also, this can be used as a basis for the development of in-service training programs.

\section{CONFLICTS OF INTEREST}

The author declare that there are no conflicts of interest in the publication of this study.

\section{SOURCE OF FUNDING:}

None. 


\section{REFERENCES}

1. Betancourt JR, Green AR, Carrillo JE, AnanehFirempong O. Defining cultural competence: a practical framework for addressing racial/ethnic disparities in health and health care. Public Health Rep. 2003; 118(4):293-302.

2. Frieden TR. Forward: CDC Health Disparities and Inequalities Report - United States, 2011. Centers for Disease Control and Prevention (CDC). MMWR Suppl, 60(1):1-2.

3. Soriano G, Aranas FC, Tejada RS. Caring behaviors, spiritual and cultural competencies: A Holistic Approach to Nursing Care. Bedan Res J. 2019; 4, 98115.

4. Jeffreys MR. Teaching Cultural Competence in Nursing and Health Care. 2nd edition. New York, NY, USA: Springer; 2010.

5. Loftin C, Hartin V, Branson M, Reyes H. Measures of Cultural Competence Nurses: An Integrative Review. The Scientific World J. 2013

6. Murphy K. The importance of cultural competence. Nurs Incredibly Easy. 2011; 9 (2), 5

7. Campinha-Bacote, J. The Process of Cultural Competence in the Delivery of Healthcare Services: a model of care. J Transcult Nurs. 2002; 13(3): 181-184

8. Rechel B, Mladovsky P, Ingleby D, Mackenbach JP, McKee M. Migration and health in an increasingly diverse Europe. The Lancet. 2013; 381(9873): 12351245

9. Ingram RR. Using Campinha-Bacote's process of cultural competence model to examine the relationship between health literacy and cultural competence $\mathbf{J}$ Adv Nurs. 2011; 68(3): 695-704

10. WHO. Global strategic directions for strengthening nursing and midwifery 2016 2020. 2016. [cited August 22 2020] Availabel from: https://www.who.int/hrh/nursing_midwifery/globalstrategic-midwifery2016-2020.pdf

11. Ghiyasvandian S, Zakerimoghadam M, Peyravi H. Nurse as a facilitator to professional communication: a qualitative study. Glob. J. Health Sci. 2014; 7(2): 294303

12. American Association of Colleges of Nursing [Internet]. Cultural competency in baccalaureate nursing education. Washington, DC: ANA; 2008 [cited June 22 2020]. Available from http://www.aacn.nche.edu/leadinginitiatives/education resources/competency.pdf

13. Shen Z. Cultural competence models and cultural competence assessment instruments in nursing: a literature review. J. Transcult. Nurs. 2015; 26(3): 308321

14. Emami A, Safipour J. Constructing a questionnaire for assessment of awareness and acceptance of diversity in healthcare institutions. BMC Health Serv Res. 2013; 13: 145 .

15. Fitzgerald EM, Cronin SN, Campinha-Bacote J. Psychometric testing of the Inventory for Assessing the Process of Cultural Competence among Healthcare
Professionals: Student version (IAPCC-SV). J of Theory Constr Test. 2009; 13: 64-68

16. Perng SJ, Watson R. Construct validation of the nurse cultural competence scale: A hierarchy of abilities. Journal of Clinical Nursing. 2012; 21: 1678-1684.

17. Gorsuch RL. Factor analysis (2nd Ed.). New Jersey, USA: Lawrence Erlbaum; 1983

18. Ferketich S. Focus on psychometrics. Aspects of item analysis. Res Nurs Health. 1991; 14(2), 165-168.

19. Polit D, Beck C. Essentials of nursing research: Appraising evidence for nursing practice. Philadelphia, PA: Wolters Kluwer Health; 2014

20. Li HC, Lopez V. Development and validation of a short form of the Chinese version of the State Anxiety Scale for Children. Int J Nurs Stud 2007; 44(4): 566-73

21. Tabachnick BG, Fidell LS. Using Multivariate Statistics (5th Ed.) Boston, MA: Pearson Education. Inc; 2007

22. Burns N, Grove SK. The practice of nursing research appraisal, synthesis, and generation of evidence (6th Ed.). St. Louis, MO: Saunders; 2009

23. Gözüm S, Tuzcu A, Kirca N. Validity and Reliability of the Turkish Version of the Nurse Cultural Competence Scale. J Transcult Nursing. 2016; 27(5), 487-495

24. Cruz JP, Colet PC, Bashtawi MA, Mesde JH, Cruz CP Psychometric evaluation of the Cultural Capacity Scale Arabic version for nursing students. Contemp Nurs. 2016; 53(1): 13-22

25. Cruz JP, Machuca Contreras FA, Ortiz López JE., Zapata Aqueveque CA, Vitorino LM. Psychometric assessment of the cultural capacity scale Spanish version in Chilean nursing students. Int Nurs Rev. 2017; 65(2): 262-269. 